\title{
Acquisition and Fast Analysis of Multi-Dimensional STEM Data
}

\author{
Andrew R. Lupini ${ }^{1}$, Albina Y. Borisevich ${ }^{1}$, Juan Carlos Idrobo ${ }^{2}$
}

${ }^{1}$ Materials Science \& Technology Division, Oak Ridge National Laboratory, Oak Ridge, TN 37831

${ }^{2}$ Center for Nanophase Materials Sciences, Oak Ridge National Laboratory, Oak Ridge, TN 37831

Scanning transmission electron microscopy (STEM) has recently undergone an exciting transition to a regime where the entire instrument can be computer controlled and the detectors now produce a data stream far faster than a human operator can interpret or analyze. Thus automated or online data analysis is becoming increasingly feasible and will be increasingly required.

One persistent challenge in aberration-corrected STEM is to accurately align the aberrationcorrector and perhaps other electron-optical elements [1]. To fully automate microscope control requires the ability to quantify the fitness of a particular condition. Thus rapid measurement of the imaging imperfections (the aberration function) constitutes a technologically relevant example. This problem has several immediate practical uses and, significantly, most of the methods currently used for this purpose can best be represented by their action on a multi-dimensional dataset, illustrating how such multi-dimensional datasets provide a novel perspective from which to consider traditional imaging modes.

As a specific example, it was recently shown that such datasets can be used to rapidly measure image aberrations [2]. This method was based on measuring the tilt-induced-shift in bright-field images acquired on pixelated detectors. However, it can also be thought of as acquiring a 4dimensional dataset, Fourier transforming over 2 of the 4 dimensions, multiplying the result by the conjugate of the transform of the central (axial) image, reverse transforming, and performing a least-squares fit to positions of local maxima. Expressed in this manner, it becomes clear that many microscope alignments are simply a processing problem and thus there exists great potential to accelerate such schemes. Recent instrumentation and software advances now provide unprecedented access to the underlying microscope control systems, presenting opportunities to control the instrument in or near real-time, faster than achievable by a human operator.

Figures 1 and 2 illustrate the 'extra' information available in these datasets with an example of analyzing the 4D dataset consisting of the scattered distribution from a single layer of graphene. The Fourier transform of the dataset can be represented in such a manner as to closely resemble the transform of a single 2D image (Fig. 1), however the phase contains additional information $[3,4]$. This information can be particularly useful for analyzing aberrations (Fig. 2), or for extracting otherwise inaccessible details, such as local magnetic properties [5]. [6]

References:

[1] O.L. Krivanek, N. Dellby \& A.R. Lupini, Ultramicroscopy 78 (1999) 1-11.

[2] A.R. Lupini, M. Chi \& S. Jesse, Journal of Microscopy 263 (2016) 43-50.

[3] J. M. Rodenburg, B. C. McCallum and P. D. Nellist, Ultramicroscopy, 48 (1993) 304. 
[4] T.J. Pennycook, et al, Ultramicroscopy, Ultramicroscopy 151 (2015) 160-167.

[5] J.C. Idrobo, et al, Advanced Structural and Chemical Imaging 2:5 (2016).

[6] Research supported by Division of Materials Sciences and Engineering Division, Office of Basic Energy Sciences, U.S. DOE (ARL, AYB) and by Oak Ridge National Laboratory's Center for Nanophase Materials Sciences (CNMS), which is sponsored by the Scientific User Facilities Division, Office of Basic Energy Sciences, U.S. Department of Energy (JCI).
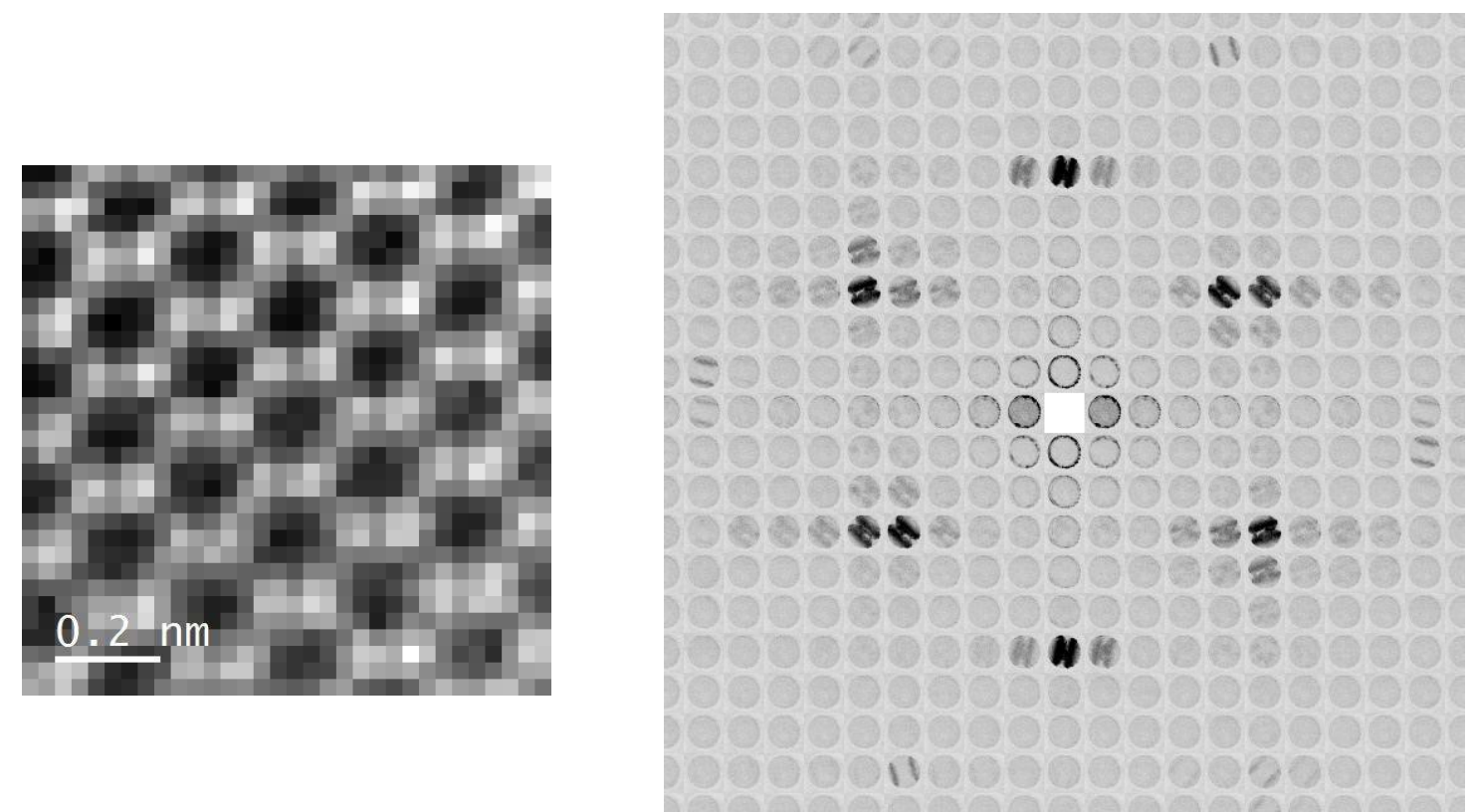

Figure 1. (Left) Annular dark field image acquired at the same time as the multi-dimensional data. Individual carbon atoms are just resolved. (Right) The amplitude of the Fourier transform of the 4D data closely resembles the Fourier transform of a single image, however, each Fourier spot is now a $2 \mathrm{D}$ plane.
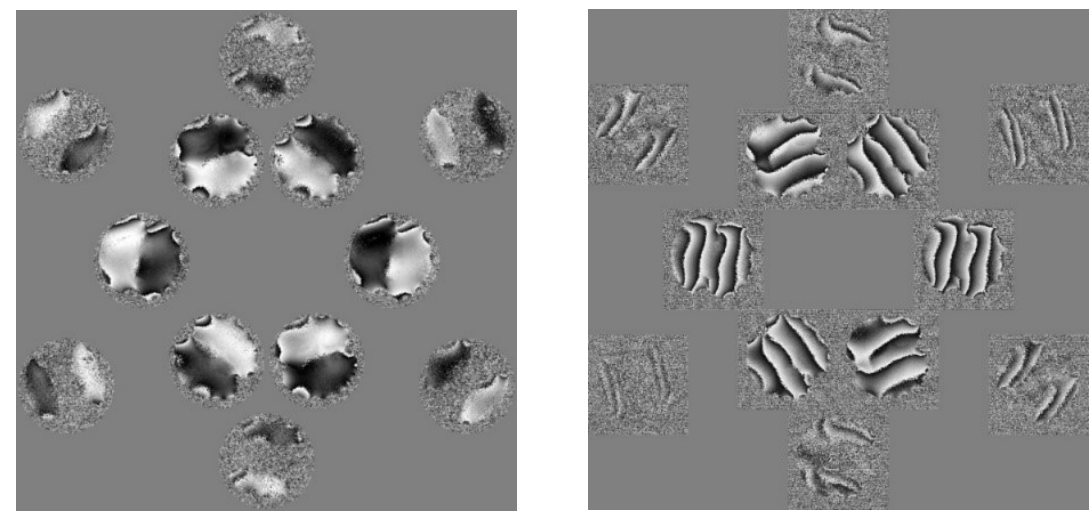

Figure 2. The phase of the Fourier transform of the data contains information about the phase of the electron beam at the detector. Selected planes from two example cases are shown. (Left) A near optimized beam displays a very uniform phase. (Right) Deliberately adding a few nanometers of defocus introduces a phase change across the aperture, visible as stripes in the transformed data. 